\title{
CBX7 AND HOXC10 PROMOTE GASTRIC CANCER CELL PROLIFERATION AND METASTASIS THROUGH NF-KB ACTIVATION
}

\author{
PENG PENG ${ }^{1 * *}$ and XIAOYU HAN² \\ 'Endoscopy Center, Shanxi Provincial People's Hospital, \\ Taiyuan, Shanxi, 030000, China \\ ${ }^{2}$ Department of Special Ward, Shanxi Provincial People's Hospital, \\ Taiyuan, Shanxi, 030000, China
}

\begin{abstract}
Identification of prognostic biomarkers and molecular mechanisms involved in the carcinogenesis of gastric cancer will expedite the innovation of new therapeutic agents to preclude tumor recurrence and metastasis. In the present study, we made an attempt to elucidate the oncogenic role of Polycomb group (PcG) protein CBX7 (Chromobox homolog 7 positioned on chromosome 22q13.1) and homeobox protein $\mathrm{C} 10$ (HOXC10) in gastric cancer tissues and cell lines. By immunohistostaining, we showed enhanced positive staining of $\mathrm{CBX} 7$ and $\mathrm{HOXC} 10$ in gastric cancer tissues than adjacent non-cancerous tissues. In addition, our real-time quantitative PCR (RT-qPCR) analysis confirmed that the relative mRNA expression of $\mathrm{CBX} 7$ and $\mathrm{HOXC10}$ is significantly upregulated ( $>3$-fold times) in gastric cancer tissues. Consequently, the patients' cumulative survival graph revealed that $\mathrm{CBX} 7$ and $\mathrm{HOXC10}$ positive individuals have a significantly shorter life span. In the next part, we have depleted CBX7 and HOXC10 expression by siRNA transfection in gastric cancer cell lines SNU-1 and SNU-16. We found the rate of cell proliferation, migration, and clonogenic efficiencies of siRNA transfected cells were significantly reduced when compared to the non-transfected cells. One step further, we also show that CBX7 and HOXC10 regulate aforesaid functions via NF- $\mathrm{kB}$ activation. In brief, these data suggest that $\mathrm{CBX} 7$ and $\mathrm{HOXC} 10$ might play an essential role in gastric cancer tumorigenesis, and hence, the development of a new anti-cancer drug that targets $\mathrm{CBX} 7$ and $\mathrm{HOXC} 10$ would definitely limit cancer cell proliferation and metastasis effectively.
\end{abstract}

Keywords: CBX7, cell migration, gastric cancer, $\mathrm{HOXC10,NF- \kappa B} \mathrm{pathway}$

Worldwide, gastric cancer is the most common life-threatening malignancy in humans, which accounts for the second leading cause for cancer-related mortality rate $(>30 \%)$ globally $(1,2)$. In recent years, with the early diagnosis includes endoscopy and advance treatment strategies the average survival of patients remains 5 years at metastatic stage (3). According to the proposed cancer stem cell theory, a minor population of cells with tumor-initiating and self-renewal properties is termed cancer stem cells (CSCs) which play a major role in tumorigenesis, invasion, angiogenesis, metastasis, and tumor recurrence (1-4). Several studies have been reported the isolation and characterization of such CSCs from gastric cancers (5-7). Nevertheless, the underlying cause for gastric cancer carcinogenesis is not well characterized so far. Polycomb group (PcG) proteins belong to the family of epigenetic regulators involved in cell proliferation and cancer progression (7), which is often found to be deregulated in different cancers. PcG proteins execute their functions at various sites in chromosomes by the organization of polycomb repressive complexes (PRCs), multi-functional protein complexes which can able to modify chromosome structure and gene expressions through epigenetic regulation (8). The implications of dysregulated PcG proteins in carcinogenesis have been reported in several tumors and therefore PcG proteins were categorized as an oncogene $(8,9)$.

Recently, the PcG protein Bmi-1 was reported as an oncogene, has an essential role in gastric cancer progression (9). Similarly, $C B X 7$ encodes for chromobox protein $\mathrm{CBX} 7$ belongs to PcG family performs a similar function as that of Bmi-1 where it impedes cell mortality and promotes cell proliferation through INK4a/ARF downregulation (9-11). Studies showed that overexpression of $\mathrm{CBX} 7$ was associated with poor prognosis, lymph node metastasis, and poor clinical outcome of gastric cancer. It has been well

\footnotetext{
* Corresponding author: e-mail: ppeng1211@163.com

\# These two authors contributed to this work equally
} 
documented that $\mathrm{CBX} 7$ overexpression has a potential oncogenic role in the progression of gastric cancer and regulates the stemness phenotype of gastric cancer stem cells. Meanwhile, decreased CBX7 expression was found to be associated with an aggressive phenotype of the thyroid, colon, and pancreatic cancers (1214). Hence, based on cellular circumstances and cancer perspective $\mathrm{CBX} 7$ protein may function either as an oncogene or tumor suppressor. Consequently, it's a pressing requirement for the researchers to clarify these controversial expression patterns of $\mathrm{CBX} 7$ in gastric cancer and its function in cancer development.

In addition, PcG proteins are also involved in the regulation of homeotic genes (HOX) expression, which has been frequently deregulated in different cancers. HOXC10 belongs to the family of HOX genes which regulates a wide array of processes such as cell proliferation, differentiation, motility, and apoptosis. In particular, HOXC10 plays a key role in tumor progression by significantly enhancing cancer cell proliferation, invasion, and metastasis and therefore $\mathrm{HOXC10}$ could be used as a biomarker for cancer prognosis (15). Elevated HOXC10 expression was often associated with metastasis and invasion of human thyroid cancer in the Cancer Genome Atlas (TCGA) dataset (16). In addition, aberrant expression of HOXC10 was observed in different types of cancers (breast, lung, and cervical) and thus confirmed the key role of HOXC10 in tumor progression (16-18). Remarkably, recent research about the role of miR-136 in gastric cancer cell line revealed that $\mathrm{HOXC10}$ acts as a promoter for peritoneal metastasis of gastric cancer (19). Nevertheless, precise research data elucidating the mechanistic role of HOXC10 in gastric cancer progression is still not clear. Considering all these facts, the present study was designed to examine the expression pattern of CBX7 and HOXC10 in gastric cancer tissues comparing with the adjacent non-cancerous tissues. Further, the role of these proteins in gastric cancer progression and metastasis was investigated in gastric cell lines (SNU-1 and SNU-16) by siRNA approach.

\section{EXPERIMENTAL}

\section{Gastric cancer tissues and cell lines}

Gastric cancer samples were obtained from the cancer patients $(n=50)$ during surgery at Endoscopy Center, Shanxi Provincial People's Hospital according to the ethical rules approved by the ethical committee of Shanxi Provincial People's Hospital, Taiyuan, Shanxi, China. At the same time, oral informed consent was also obtained from patients. No patients' details and consents are included in this manuscript as per the hospital rules. Clinicopathological features of obtained gastric cancer samples: Origin: adenocarcinoma; Cancer stage: III \& IV; Differentiation: moderate to well. Adjacent non-cancerous tissues were also collected at the same time, used as control. Human gastric cancer cell lines SNU-1 and SNU-16 were obtained from the Chinese Academy of Sciences, Shanghai. Cell culturing was performed in RPMI1640 medium provided with appropriate antibiotics and $10 \%$ fetal bovine serum (FBS).

\section{Immunohistochemical staining}

Human gastric cancer tissue sections (approximately $4 \mu \mathrm{M}$ thickness) were paraffin-embed as per the described procedure (20). Tissue slides were incubated with Polyclonal anti-HOXC10 antibody (1 : 500; Santa Cruz Biotechnology) and polyclonal anti-CBX7 at $4^{\circ} \mathrm{C}$ for overnight (1: 500; Santa Cruz Biotechnology). Followed by overnight incubation, cells were counterstained with secondary antibody conjugated with HRP (horseradish peroxidase). Finally, cells were viewed under a confocal microscope (Olympus, Tokyo, Japan) and the images captured were processed by Image J.

\section{RNA extraction and $q R T-P C R$ analysis}

Trizol reagent was used to extract total cellular RNA from tissues and cells as per the manufacturer's protocol. Fermantas Kit was used for preparing complementary DNA synthesis (cDNA) and subsequently, $1 \mu \mathrm{L}$ of cDNA was mixed with IQ Supermix and SYBRGreen reagents to perform real-time quantitative PCR Bio-Rad-iCycler IQ detection system. The following forward $(\mathrm{F})$ and reverse $(\mathrm{R})$ primers were used for amplification: HOXC10: F - ACA TCT GGA ATC GCC TCA GC and R - GGC TCT GCT CCG TCT TGA TT (15); $C B X 7$ : F - FTGGGTTTCGGACCTCTCTT and R - CGTCATGGCCTACGAGGA; GAPDH: F - AAT GAA GGG GTC ATT GAT GG and R AAG GTG AAG GTC GGA GTC AA (21). The PCR parameters used for 35 cycles were: $94^{\circ} \mathrm{C}$ for $30 \mathrm{~s}$, $60^{\circ} \mathrm{C}$ for $45 \mathrm{~s}$, and $72^{\circ} \mathrm{C}$ for $30 \mathrm{~s}$. The DNA amplicons were visualized on $1 \%$ agarose gel electrophoresis by ethidium bromide staining. The band intensity was measured with Image J software (2.0) and normalized with GAPDH, the housekeeping gene. The relative mRNA expression for each gene is calculated and expressed by the $2-\Delta \Delta \mathrm{Ct}$ method.

\section{siRNA transfection}

Approximately, $2 \times 10^{6}$ Cells were cultured in 6 well plates for $24 \mathrm{~h}$. SiRNA oligos of $20 \mathrm{nM}$ were mixed with $6 \mu \mathrm{L}$ of Lipofectamine ${ }^{\circledR} 2000$ (Invitrogen) in RPMI medium and incubated for $15 \mathrm{~min}$ at room 
temperature. Subsequently, the reaction mix was gently added to the cells and incubated for $24-48 \mathrm{~h}$ at $37^{\circ} \mathrm{C}$. The siRNA sequences used in this study were: HOXC10: GCGCAGGCAUGUAUAUGCATT (21); CBX7 siRNA (sc-72816) and scramble RNAi (control) were purchased from Santa Cruz Biotechnology and Thermo Fishers, respectively. After $24 \mathrm{~h}$ incubation of RNAi transfection, cells were harvested and subjected to western blot, RT-PCR, and other in vitro cellular assays.

\section{Luciferase assay}

Cells were cultured in 12 well plates $\left(10^{5}\right.$ cells/ well) for $24 \mathrm{~h}$ and transfected with the plasmids according to the manufacturer's protocol: $100 \mathrm{ng}$ of NF-kB reporter plasmid (BPS Bioscience Kit), $100 \mathrm{ng}$ of control luciferase plasmid, plus $5 \mathrm{ng}$ pRL-TK Renilla plasmid which acts as a control (Promega). Luciferase activity was measured by using Promega Dual Reporter Assay kit and the relative luciferase activity was estimated by the ratio of NF-kB reporter luciferase activity to the transfection control. The relative NF-kB activity was estimated by NF-kB Reporter kit (BPS Bioscience).

\section{Western blot analysis}

Cells were washed in ice-cold PBS and lysed with 2X Laemmli sample buffer (Biorad). Cell lysates were prepared and the proteins were separated by $10 \%$ sodium dodecyl sulfate-polyacrylamide (SDS-PAGE) gel electrophoresis. Further, the proteins were transferred to nitrocellulose membrane and blocked with blocking buffer (5\% skim milk powder in TBST). Subsequently, the membrane is incubated with primary antibodies: polyclonal rabbit anti-HOXC10 (1 : 1000; Sigma), monoclonal rabbit anti-ATM (1 : 1000; Cell Signaling Technology), polyclonal anti-CBX7 (1 : 1000; Santa Cruz Biotechnology), and monoclonal mouse anti-a-tubulin (1 : 4000; Sigma). The secondary antibody used was a mouse or rabbit Anti- IgG (1 : 1000; Santa Cruz Biotechnology) and the protein signal was detected by an enhanced chemiluminescence kit (Amersham Pharmacia Biotech). The protein band intensity was quantified by using image analysis software and the values presented in the graph are the average values from the three independent blots.

\section{In vitro cellular assays \\ Migration assay}

Cells cultured $\left(2 \times 10^{6}\right.$ Cells $)$ in invasion chambers (BD matrigel chambers) for $48 \mathrm{~h}$ were subsequently swabbed to the top layer with a matrigel Q-tip to wash off the non-migrated cells. Hematoxylin staining was performed to stain the invading cells which are present on the membrane, mounted on the slides, and cells were visualized under a microscope at a $40 \mathrm{X}$ objective lens. The number of migrated cells was counted and the values represented in the graph are from three independent experiments.

\section{Cell proliferation assay}

Cells were cultured in 96-well plates $\left(10^{6}\right.$ cells/ well) and the rate of cell growth was evaluated by measuring the optical density (OD) at $450 \mathrm{~nm}$. Cells were treated with $10 \mu \mathrm{L}$ of Cell Counting Kit-8 (CCK-8; Sigma) $3 \mathrm{~h}$ before the measurement of OD at $450 \mathrm{~nm}$. The estimated rate of cell proliferation was represented as a quantification graph from three independent experiments.

\section{Colony formation assay}

Approximately, $5 \times 10^{2}$ cells/well were cultured in 6-well plates for two weeks. Subsequently, plates were washed gently with PBS, and the colonies formed were fixed with $4 \%$ paraformaldehyde (PFA) and counterstained with $0.1 \%$ crystal violet solution, and the colonies were counted.

\section{Apoptosis assessment}

Cells grown for $24 \mathrm{~h}$ were harvested and fixed icecold methanol. Following the fixation propidium iodide (PI from Sigma-Aldrich) staining was performed overnight in a $37^{\circ} \mathrm{C}$ water bath at dark conditions. The rate of apoptosis was evaluated by flow cytometry (BD Bioscience USA). The percentage of apoptotic cells between the siRNA transfected and non-transfected cells were calculated from dot plot analysis of FACs profile and they were expressed as quantitative data. The values represented in the graph are the average values from the three independent experiments.

\section{Statistical analysis}

SPSS 15.0 software was used to perform statistical analyses. Kaplan-Meier method was employed to make the patients Cumulative survival curves and a Log-rank test was used for univariate analysis. Student's T-Test and one-way analysis of variance (ANOVA) were performed to compare the difference between the two groups. The data represented in the graphs were mean $\pm \mathrm{SD}$ and the $\mathrm{P}$ value less than 0.05 was considered as statistically significant.

\section{RESULTS AND DISCUSSION}

\section{CBX7 and HOXC10 overexpression in gastric cancer tissues}

Several studies have been reported the role of PcG family of proteins in carcinogenesis and 
one amongst is Bmi-1, function as an oncogene in many cancers (8-10). Recently, it was found that CBX7 shares the characteristic features of Bmi-1 and performs the same mechanistic role in limiting cellular senescence and promoting cancer progression. The data concerning the detailed function of CBX7 in different cancers remains scanty and especially in gastric cancer, it's not well characterized so far. Meanwhile, homeotic genes (HOX) are also regulated by $\mathrm{PcG}$ proteins, amongst $\mathrm{HOXC10}$ elevated expression was frequently observed in different cancers (11-13). Similar to CBX7, only a few reports available describing the oncogenic role of HOXC10 in gastric cancer. But the extended role and underlying molecular mechanism of HOXC10 in gastric cancer progression are still lagging. Hence, in the present study, we have analyzed the expression pattern of $\mathrm{CBX} 7$ and $\mathrm{HOXC10}$ in primary gastric cancer tissues by immunohistochemistry (IHC). The paraffin-embedded cancer biopsies $(>80 \%$ of samples) showed intense positive staining to $\mathrm{CBX} 7$ and
HOXC10. They are mostly localized into the nucleus and showed higher expression in gastric cancer tissues. However, no CBX7 positive and very little HOXC10 staining were observed in adjacent noncancerous tissues (Figure 1A). Further, the quantitative-PCR results revealed that mRNA expression levels of CBX7 and HOXC10 are significantly $(p<0.01)$ upregulated in gastric cancer tissues when compared to non-cancerous tissues. The rate of mRNA transcription was 3-fold times higher in gastric cancer tissues (Figure 1B). Overexpression of CBX7 was frequently associated with an aggressive phenotype in B-cell lymphomas of transgenic mice and also influence the survival rate of mouse embryonic fibroblasts (22-24). Therefore, CBX7 functions as an oncogene like Bmi-1. Conversely, loss of CBX7 was allied with the development of aggressive malignant phenotype in thyroid, colorectal and pancreatic cancers. Based on these findings, researchers proposed that $\mathrm{CBX} 7$ functions as a tumor suppressor.
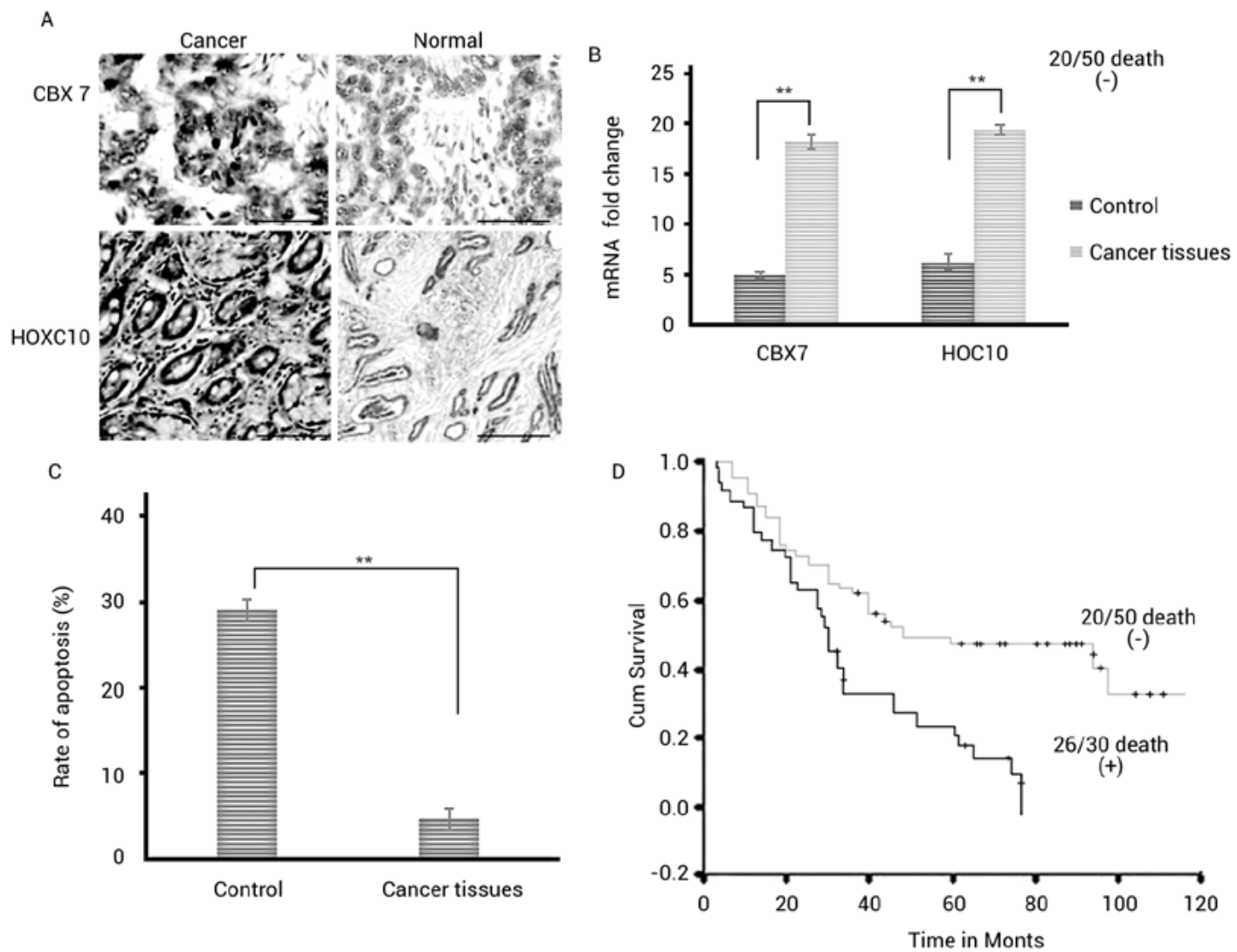

Figure 1. Increased expression of $\mathrm{CBX} 7$ and $\mathrm{HOXC} 10$ in gastric cancer tissues. A. Representative microscopic pictures for immunohistostaining of CBX7 and HOXC10 in human gastric cancer and control tissues. Magnifications: Upper panel: 400×; lower: 40×. Scale bar: Upper panel - $50 \mu \mathrm{m}$; lower - $200 \mu \mathrm{m}$. B. Quantitative PCR analysis of CBX7 and HOXC10 gene transcription in gastric cancer tissues compared with control tissues $(n=50 ; * * p<0.01)$, GAPDH was used for normalization. C. Evaluation rate of apoptosis between control and gastric cancer tissues, $* * \mathrm{p}<0.01$. D. Kaplan-Meier survival graph explaining patients cumulative survival in months based on $\mathrm{CBX} 7 / \mathrm{HOXC10}$ positive and negative expression. 
In addition, apoptosis was significantly inhibited in gastric cancer biopsies rather than control tissues (Figure 1C). Consequently, our patients' survival data suggest that the death rate of patients who were positive to both $\mathrm{CBX} 7$ and HOXC10 was significantly $(\mathrm{p}<0.01)$ higher than that of negative individuals (Figure 1D). The median monitoring time was set to 54 months and the number of patients who died in this time frame was around 45 . In consistence with our findings, earlier reports showed that elevated expression of $\mathrm{CBX} 7$ and $\mathrm{HOXC10}$ in gastric cancer tissues influences patients' survival rate $(8,14)$. Taken together, these data suggest that overexpression of CBX7 and HOXC10 are highly associated with poor prognosis and survival rate of gastric cancer patients. Also, CBX7 and HOXC10 would be valuable cancer biomarkers or prognostic factors for the diagnosis of gastric cancer.

\section{Increased expression of $\mathrm{CBX} 7$ and HOXC10 contributes malignant phenotype of gastric cancer}

In order to determine the impact of $\mathrm{CBX} 7$ and HOXC10 knockdown on gastric tumorigenesis, we have performed in vitro cellular assays by using

A

\section{CBX7 RNAi}

$(-)$

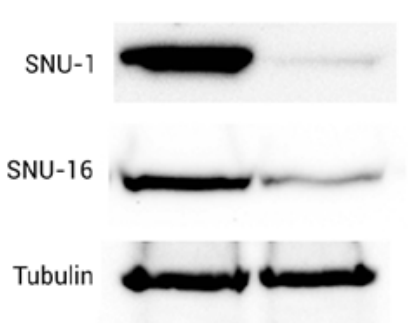

C

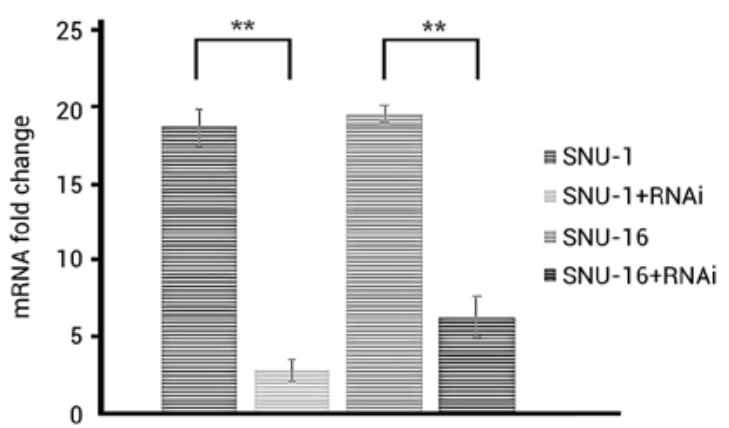

gastric cell lines SNU-1 and SNU-16 upon silencing CBX7 and HOXC10. By RNA interference (RNAi) approach, the $\mathrm{CBX} 7$ and $\mathrm{HOXC10}$ levels were depleted in the aforesaid cell lines and subjected to in vitro cellular assays in order to determine their function in gastric cancer carcinogenesis. Western blot data confirmed that siRNA transfected cells showed reduced expression of $\mathrm{CBX} 7$ (Figure 2A) and HOXC10 (Figure 2B) than non-transfected cells. In addition, the relative $\mathrm{mRNA}$ expression of $\mathrm{CBX} 7$ and HOXC10 was significantly downregulated in siRNA transfected cells (Figures 2C and 2D). With these confirmatory results, we have proceeded with the siRNA transfected cells to the next level of experiments. Interestingly, the proliferation rate of CBX7 and HOXC10 gene silenced cells were gradually declined from day 1 to day 3 . These significant differences were observed in both the gastric cancer cell lines (Figures 3A and 3B). We speculate this might be due to the cell cycle arrest at G0/G1 phase and hence the growth was inhibited. Similarly, it was reported that $\mathrm{CBX} 7$ stable knockout leads to induction of cellular senescence and inhibits the oncogenesis process by limiting cell proliferation (25). Subsequently, colony formation assay unveiled HOXC10 RNAi

$(-)$

$(+)$

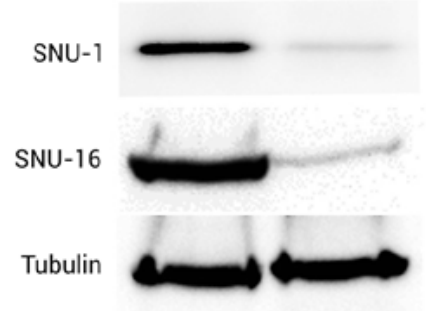

D

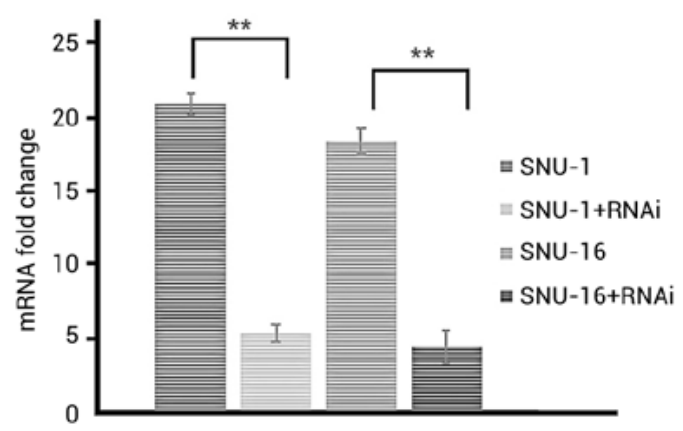

Figure 2. CBX7 and HOXC10 depletion in SNU-1 and SNU-16 cell lines. A \& B. Western blot results showing decreased protein expression of CBX7 and HOXC10 upon siRNA transfection. C \& D. Quantification graph from qPCR analysis displaying transcriptional downregulation of CBX7 and HOXC10 in siRNA transfected cells; **p $<0.01$. 
A

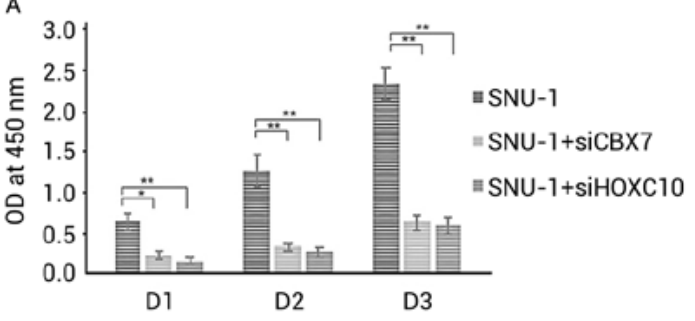

C

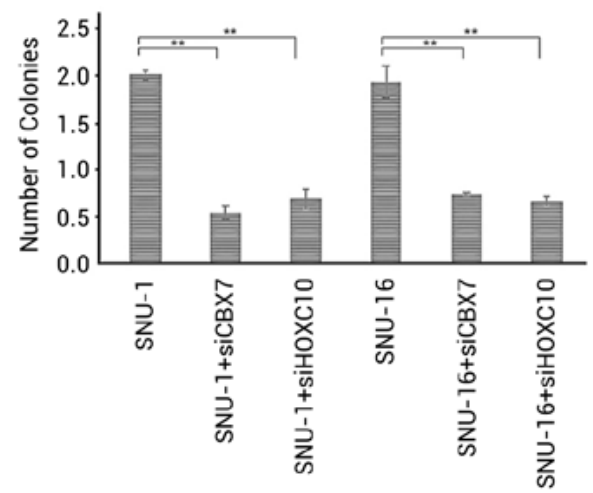

B

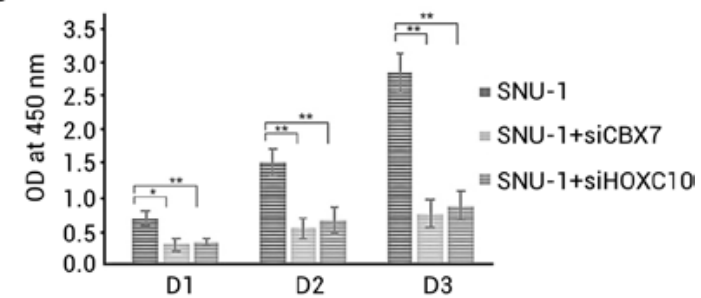

C

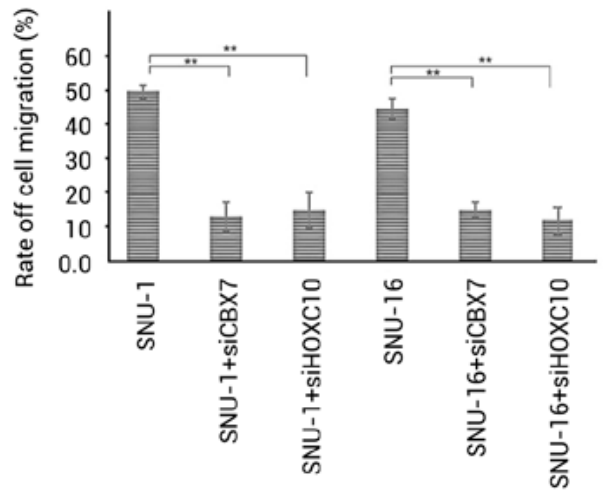

Figure 3. Overexpression of $\mathrm{CBX} 7$ and $\mathrm{HOXC10}$ contributes to gastric cancer carcinogenesis. Cell proliferation assay (A \& B) showing that the proliferation rate of siRNA transfected cells was limited significantly compared to corresponding control cells. C. Colony formation assay graph displaying that less number of colonies were formed by RNAi transfected cells. D. The rate of cell motility was significantly slower in $\mathrm{CBX7}$ and HOXC10 silenced cells. $(* \mathrm{p}<0.05 ; * * \mathrm{p}<0.01)$

that the total number of colonies formed by siRNA CBX7 and HOXC10 cells is significantly less than the non-transfected cells (Figure 3C). It was also noted that colonies formed by siRNA transfected cells are smaller and grow slower, whereas the colonies formed by control cells are larger and have faster growth. In addition, the rate of cell migration was significantly $(\mathrm{p}<0.01)$ decreased in SNU-1 and
A

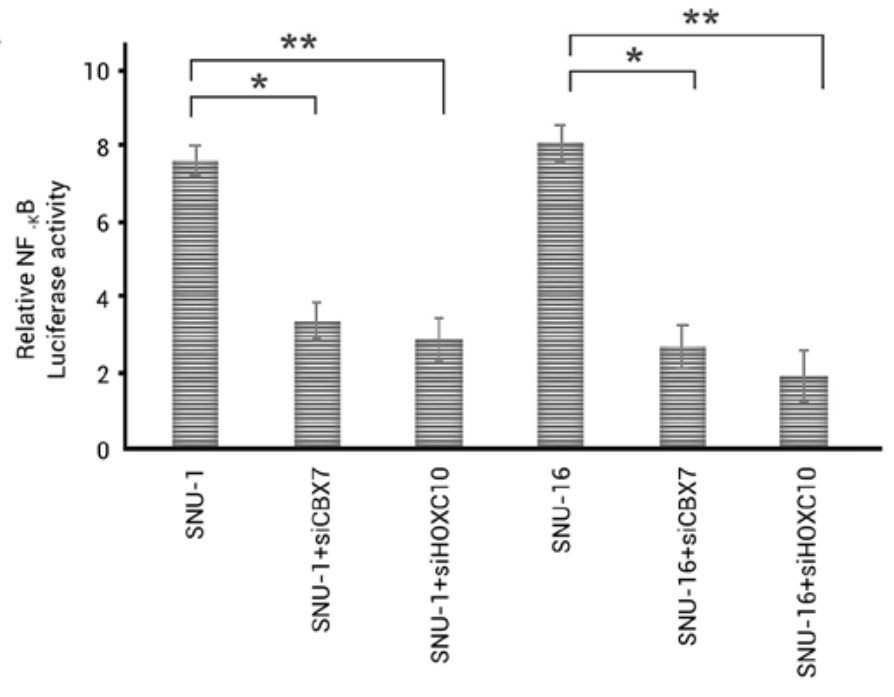

SNU-1

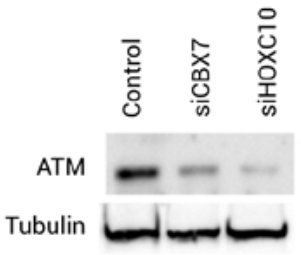

SNU-16

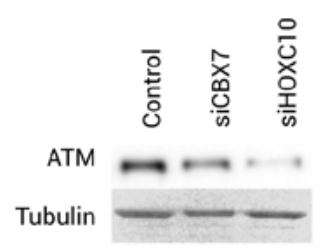

Figure 4. $\mathrm{CBX} 7$ and $\mathrm{HOXC10}$ activate ATM/NF-kB signaling pathway. A. Dual-luciferase reporter assay showing significantly suppressed NF-kB transcriptional activity upon siRNA transfection in SNU-1 and SNU-16 cells, ${ }^{* *} p<0.01$. B. Western blot showing reduced ATM expression in RNAi cells when compared to corresponding control cells. 
SNU-16 cells upon silencing of $\mathrm{CBX} 7$ and $\mathrm{HOXC10}$ (Figure 3D). In line with these findings, $\mathrm{CBX} 7$ and HOXC10 with miR-136 demonstrated that dramatically decreased migration and invasion potential of gastric cancer cells $(14,15)$. Consequently, the rate of migration of siRNA transfected cells was greatly reduced. In summary, data from SNU-1 and SNU-16 cell lines suggest the oncogenic role of $\mathrm{CBX} 7$ and $\mathrm{HOXC10}$ in gastric progression and metastasis.

\section{CBX7 and HOXC10 promote gastric cancer carcinogenesis via $\mathrm{NF}-\mathrm{kB}$ activation}

Recent studies proposed that the stemness and invasion properties of gastric cancer cells are regulated by $\mathrm{CBX} 7$ and $\mathrm{HOXC10}$, respectively. At the same time, their functions are executed through the activation of AKT-NF- $\kappa$ B-miR-21 pathways $(9,15)$. In the present study, by luciferase reporter assay we found that NF- $\kappa$ B transcriptional regulation was interrupted and its transcriptional activity was dramatically decreased upon $\mathrm{CBX} 7$ and HOXC10 depletion (Figure 4A). As it was already documented that ATM signaling is required for the NF-kB activation and there is a positive correlation exist between the ATM protein expression and NF- $\kappa \mathrm{B}$ activation (15). Subsequently, the western blot analysis (Figure 4B) showing the altered expression of ATM in siRNA transfected cells. Thus, NF-kB activation was directly proportional to the ATM signaling and the decreased ATM expression might be the cause for NF- $\kappa \mathrm{B}$ downregulation in siRNA transfected cells $(26,27)$. Ultimately, these results demonstrated the involvement of NF-kB and ATM signaling in gastric cancer proliferation, migration, and invasion which was regulated by $\mathrm{CBX} 7$ and HOXC10. Future research regarding double knockout of $\mathrm{CBX} 7$ and HOXC10 in animal models or CRISPR gene editing cell lines would certainly provide more mechanistic information for the development of a new therapeutic agent with precise targeting information.

\section{CONCLUSIONS}

In summary, our study reveals that $\mathrm{CBX} 7$ and HOXC10 are overexpressed in gastric cancer tissues and enhances gastric cancer malignant phenotype. Further, $\mathrm{CBX} 7$ and $\mathrm{HOXC10}$ downregulation by siRNA approach in gastric cancer cell lines in SUN-1 and SNU-16 compromise the gastric cancer progression by significantly limiting cell proliferation, migration, and invasion. Finally, our data showed that $\mathrm{CBX} 7$ and HOXC10 exert their oncogenic role in gastric cancer progression via activation of NF- $\kappa$ B pathway. Therefore, future research is a pressing need for determining the molecular mechanism of CBX7 and HOXC10 activation and its regulation will provide more insights for the development of agents such as HOXC10 inhibitors, or small molecule inhibitors (for anti-cancer treatment) and the development of targeted therapies to provide better treatment outcome.

\section{Conflict of interest}

The authors declared no conflict of interest.

\section{REFERENCES}

1. Ni S.J., Zhao L.Q., Wang X.F., Wu Z.H., Hua R.X., et al.: J. Hematol. Oncol. 11, 17 (2018).

2. Li K., Dan Z., Nie Y.Q.: World J. Gastroenterol. 20, 5420 (2014).

3. Bickenbach K., Strong V.E.: J. Gastric. Cancer. 12, 55 (2012).

4. Di Croce L., Helin K.: Nat. Struct. Mol. Biol. 20, 1147 (2013).

5. Takaishi S., Okumura T., Tu S., Wang S.S., Shibata W., et al.: Stem Cells 27, 1006 (2009).

6. Xue Z., Yan H., Li J., Liang S., Cai X.: J. Cell. Biochem. 113, 302 (2012).

7. Gil J., Bernard D., Peters G.: DNA Cell Biol. 24, 117 (2005).

8. Zhang X.W., Zhang L., Qin W., Yao X.H., Zheng L.Z., et al.: J. Exp. Clin. Cancer. Res. 29, 114 (2010).

9. Zhang X.W., Sheng Y.P., Li Q., Qin W., Lu Y.W., et al.: Mol. Cancer 9, 40 (2010).

10. Guo W.J., Datta S., Band V., Dimri G.P.: Mol. Biol. Cell 18, 536 (2007).

11. Pallante P., Federico A., Berlingieri M.T., Bianco M., Ferraro A., et al.: Cancer Res. 68, 6770 (2008).

12. Karamitopoulou E., Pallante P., Zlobec I., Tornillo L., Carafa V., et al.: Eur. J. Cancer. 46, 1438 (2010).

13. Pallante P., Terracciano L., Carafa V., Schneider S., Zlobec I., et al.: Eur. J. Cancer 46, 2304 (2010).

14. Guo C., Hou J., Ao S., Deng X., Lyu G.: Chin. J. Cancer Res. 29, 572 (2017).

15. Yao S., He L., Zhang Y., Ye L., Lai Y., et al.: Biochem. Biophys. Res. Commun. 501, 628 (2018).

16. Feng X., Li T., Liu Z., Shi Y., Peng Y.: Mol. Biosyst. 11, 2946 (2015).

17. Sadik H., Korangath P., Nguyen N.K., Gyorffy B., Kumar R., et al.: Cancer Res. 76, 4443 (2016). 
18. Tang X.L., Ding B.X., Hua Y., Chen H., Wu T., et al.: Front. Physiol. 8, 557 (2017).

19. Zheng J., Ge P., Liu X., Wei J., Wu G., Li X.: Tumour Biol. 39, 11 pages (2017).

20. Wang J., Wang T., Xu J., Chen W., Shi W., Cheng J., et al.: Chin. J. Cancer. Res. 28, 355 (2016).

21. Zheng X., Zhou J., Zhang B., Zhang J., Wilson J., et al.: BMC Cancer 15, 145 (2015).

22. Shi D., Liu J.: Yonsei Med. J. 59, 1166 (2018).

23. Datta S., Hoenerhoff M.J., Bommi P., Sainger R., Guo W.J., et al.: Cancer Res. 67, 10286 (2007).
24. Gil J., Bernard D., Martinez D., Beach D.: Nat. Cell Biol. 6, 67 (2004).

25. Dimri G.P.: Cancer Cell 7, 505 (2005).

26. Huang T.T., Wuerzberger-Davis S.M., Wu Z.H., Miyamoto S.: Cell 115, 565 (2003).

27. Sakamoto K., Hikiba Y., Nakagawa H., Hirata Y., Hayakawa Y., Kinoshita H., et al.: Oncogene 32, 1854 (2013). 